\title{
Gabor Analysis for Non-Rectangular Lattices and the Fractional Fourier-Transform
}

\author{
Preben Gråberg Nes \\ Department of Mathematical Sciences, Norwegian University of Science and Technology \\ N-7491 Trondheim, Norway \\ prebengr@math.ntnu.no
}

\begin{abstract}
In this article we study Gabor systems for non-rectangular lattices. We construct a modified Zak-transform which diagonalizes the Gabor frame operator corresponding to non-rectangular lattices at critical density. We also construct a representation of the Gabor frame operator similar to the Walnut representation. The key tool to develop these results is the fractional Fourier-transform. This approach for studying Gabor systems for non-rectangular lattices is to some extent complementary to the conventional method based on the representation theory on the Heisenberg-group.
\end{abstract}

Key words and phrases : frames, non-rectangular lattices, Gabor analysis, Walnut representation theorem, Zak-transform, fractional Fouriertransform

2010 AMS Mathematics Subject Classification — 42A38, 42C15, $94 \mathrm{~A} 12$

\section{Introduction.}

In this article we study Gabor systems for non-rectangular lattices. We obtain a modified Zak-transform for these Gabor systems, and a new representation of the Gabor frame operator similar to the classical Walnut representation. To obtain these results we have used a fractional Fourier-transform corresponding to the specific shape of the lattice.

We briefly review some important concepts from Gabor theory. For further details about this topic, see e.g. [5]. By a lattice we mean the set of points $\mathbf{A} \mathbb{Z}^{2}=\left\{\mathbf{A}\left(\begin{array}{c}k \\ n\end{array}\right): k, n \in \mathbb{Z}\right\}$ where $\mathbf{A}$ is a real and invertible $2 \times 2$ matrix. For $\lambda=(a, b)$ with $a, b \in \mathbb{R}$ the operator $\pi_{\lambda} f(t)=M_{b} T_{a} f(t)=e^{2 \pi i b t} f(t-a)$ denotes a time-frequency shift of the function $f$, where $M_{b}$ and $T_{a}$ denote the usual modulation- and translation-operator. The collection of time-frequency shifts 
$\mathcal{G}\left(g, \mathbf{A} \mathbb{Z}^{2}\right)=\left\{\pi_{\lambda} g: \lambda \in \mathbf{A} \mathbb{Z}^{2}\right\}$ is the Gabor system corresponding to the window $g \in L^{2}(\mathbb{R})$. The system is called a Gabor frame if there exist positive constants $A$ and $B$ such that;

$$
A\|f\|_{2}^{2} \leq \sum_{\lambda \in \mathbf{A} \mathbb{Z}^{2}}\left|\left\langle f, \pi_{\lambda} g\right\rangle\right|^{2} \leq B\|f\|_{2}^{2}
$$

for all $f \in L^{2}(\mathbb{R})$.

The Gabor frame operator is defined as;

$$
S_{g, g, \mathbf{A} \mathbb{Z}^{2}} f(t)=\sum_{\lambda \in \mathbf{A} \mathbb{Z}^{2}}\left\langle f, \pi_{\lambda} g\right\rangle_{L^{2}(\mathbb{R})} \pi_{\lambda} g(t), \quad f \in L^{2}(\mathbb{R}) .
$$

It is well-known that $\mathcal{G}\left(g, \mathbf{A} \mathbb{Z}^{2}\right)$ is a Gabor frame if the Gabor frame operator is bounded and has a bounded inverse. For rectangular lattices $\Lambda=a \mathbb{Z} \times b \mathbb{Z}$ the Walnut representation theorem and Zak-transform are key tools for studying boundedness and invertibility of the Gabor frame operator.

We remind that the Walnut representation has the form;

$$
S_{g, g, \Lambda} f(t)=b^{-1} \sum_{n} G_{n}(t) T_{\frac{n}{b}} f(t)
$$

where $G_{n}(t)=\sum_{k} T_{a k} g(t) T_{a k+\frac{n}{b}} \bar{g}(t)$, [19]. The correlation function $G_{n}(t)$ corresponds to time shifts of the window $g$, and is independent of $f$.

By considering the Walnut representation and the correlation-function $G_{n}$ one can study conditions on the window $g$ ensuring that the Gabor frame operator is bounded on $L^{2}(\mathbb{R}),[5$, Ch. 6]. In addition the representation can be used as a starting point for studying other central tools in Gabor analysis, such as Janssen's representation, the Wexler-Raz biorthogonality theorem, the RonShen duality principle, and the Zak-transform.

The Zak-transform diagonalizes the Gabor frame operator corresponding to rectangular lattices at critical density $a b=1$, that is $\mathcal{Z}_{a} S_{g, g} f=a \mathcal{Z}_{a} g \overline{\mathcal{Z}_{a} g} \mathcal{Z}_{a} f$. As a consequence the invertibility of the Gabor frame operator follows by properties of $\mathcal{Z}_{a} g,[2,5,7]$. Zak-transform methods have been used to verify that the windows $g(t)=(\cosh t)^{-1}$ and $g(t)=e^{-|t|}$ are Gabor frames if $0<a b<1$, and that $g(t)=\chi_{[0, \infty[} e^{-t}$ is a Gabor frame for $0<a b \leq 1,[6,8,9]$. In this article we construct generalizations of both the Zak-transform and the Walnut representation theorem to non-rectangular lattices in $\mathbb{R}^{2}$.

Gabor analysis is often studied for non-rectangular lattices through representation theory on the Heisenberg-group, [4]. Independently, one can consider Gabor systems corresponding to non-rectangular lattices using the fractional Fourier-transform. It turns out that using well-known properties of the fractional Fourier-transform allows one to study (1) on non-rectangular lattices using similar tools as in the rectangular case, such as Poisson summation formula 
and properties of Fourier-series.

The fractional Fourier-transform was first studied by V. Namias in [16], and later by e.g. [1, 14, 15, 17]. The fractional Fourier-transform is a widely recognized tool in optics $[11,12,17]$, and has in recent years become a popular tool in harmonic analysis as well $[10,18,20]$. The fractional Fourier-transform can be associated with a rotation of the time-frequency plane. In particular, for any $\theta \in(-\pi, \pi)$ there is a fractional Fourier-transform which can be interpreted as a $\theta$-rotation of the time-frequency plane.

We first introduce a modified Walnut representation theorem for vertically sheared lattices, i.e. lattices of the form $\mathbf{S} \Lambda=\{(a k, s b k+b n): k, n \in \mathbb{Z}\}, 0 \leq s<1$. For such lattices the time component is independent of frequency-shifts. In particular we will see that one can use the Poisson-summation formula and properties of Fourier-series to construct a modified Walnut representation theorem for such lattices.

The Walnut representation theorem can be generalized to arbitrary lattices in $\mathbb{R}^{2}$ by using the fractional Fourier-transform. For any $\theta \in(-\pi, \pi)$ there exists a fractional Fourier-transform which can be associated with a $\theta$-rotation of the time-frequency plane. In particular one can obtain a Walnut representation of $S_{g, g, \mathbf{R}_{\theta} \mathbf{S} \Lambda}$ by considering the fractional Fourier-transform and the Gabor frame operator associated to the lattice $\mathbf{S} \Lambda$. Let $\mathbf{R}_{\theta}$ denote the usual $2 \times 2$ rotation matrix. It is well-known that for any lattice in $\mathbb{R}^{2}$ there exists a $\theta \in(-\pi, \pi)$ and a $s \in[0,1)$ such that the lattice can be represented in the form $\mathbf{R}_{\theta} \mathbf{S} \Lambda$.

The translation- and modulation-operators commute with respect to rectangular lattices at critical density. As a consequence there exists a unitary operator which simultaneously diagonalizes these operators [3]. It is well-known that the Zak-transform provides such diagonalization, and diagonalizes the Gabor frame operator. In difference to the rectangular case, the translation and modulation operators do not commute for non-rectangular lattices. The operators can therefore not be diagonalized simultaneously. Instead we consider joint translation-modulation operators associated to the Gabor frame operator.

The suggested modified Zak-transform diagonalizes the joint translationmodulation operators, and the Gabor frame operator corresponding to any non-rectangular lattice at critical density. Using the same method as for the Walnut-representation we initially consider a modified Zak-transform for vertically sheared lattices. For such lattices one can define a modified Zak-transform in terms of a Fourier-series modulated with a Gaussian chirp. Since the fractional Fourier-transform is unitary we will see that this transform extends to any lattice in the form $\mathbf{R}_{\theta} \mathbf{S} \Lambda$. Note that a similar modified Zak-transform was suggested by van Leest and Bastiaans in the special case of the quincunx lattice $\{(a k, b k / 2+b n): k, n \in \mathbb{Z}\},[2]$.

The article is organized as follows. In the next section we remind the reader of some well-known facts about the fractional Fourier-transform. We conclude 
with considering the action of the fractional Fourier-transform on the Gabor frame operator and a Gabor system. In Sect.3 we discuss a modified Walnut formula representing the Gabor frame operator with respect to any lattice in $\mathbb{R}^{2}$. In Sect.4 we study a modified Zak-transform. We will see that the modified Zak-transform shares most of the properties of the usual Zak-transform.

\section{The fractional Fourier-transform.}

The fractional Fourier-transform corresponding to the angle $\theta \in(-\pi, \pi)$ is defined as;

Definition 1 (Fractional Fourier-transform, [17]). Let $f \in L^{1}(\mathbb{R})$ and $0<|\theta|<\pi$. The fractional Fourier-transform $\mathcal{F}_{\theta}(f)(\xi)=f_{\theta}(\xi)$ is defined as;

$$
\mathcal{F}_{\theta}(f)(\xi)=A_{\theta} \int_{\mathbb{R}} f(t) e^{-\frac{2 i \pi \xi t}{\sin \theta}+\frac{i \pi\left(\xi^{2}+t^{2}\right) \cos \theta}{\sin \theta}} d t,
$$

where $A_{\theta}=\frac{e^{-\frac{i}{2}\left(\frac{\pi}{2} \operatorname{sgn} \theta-\theta\right)}}{\sqrt{|\sin \theta|}}$.

The fractional Fourier-transform is well-defined for functions in $L^{p}(\mathbb{R})$ for $1 \leq p \leq 2$ and is a unitary operator on $L^{2}(\mathbb{R})$, see e.g. Cor. 1 . If $\theta=0$ the fractional Fourier-transform equals the identity-operator, and if $\theta= \pm \frac{\pi}{2}$ the corresponding operator $\mathcal{F}_{\theta}$ equals respectively the Fourier- and inverse Fouriertransform.

The classical Fourier-transform satisfies the relation;

$$
\mathcal{F}\left(M_{b} T_{a} f\right)=e^{2 \pi i a b} M_{-a} T_{b} \mathcal{F}(f) .
$$

Note that the time-frequency shift $\pi_{(a, b)}$ is - up to the unimodular factor $e^{2 \pi i a b}$ - transformed to the time-frequency shift $\pi_{(b,-a)}$. One can therefore interpret the Fourier-transform as a $-\frac{\pi}{2}$-rotation of the time-frequency plane. The similar relation for the fractional Fourier-transform has the form;

Lemma 1 (Modulation and translation, [17]). Let $0<|\theta|<\pi$ and consider $a, b \in \mathbb{R}$. The fractional Fourier-transform satisfies;

$$
\begin{aligned}
\mathcal{F}_{\theta} T_{a} & =e^{-i \pi a^{2} \sin \theta \cos \theta} T_{a \cos \theta} M_{-a \sin \theta} \mathcal{F}_{\theta}=e^{i \pi a^{2} \sin \theta \cos \theta} M_{-a \sin \theta} T_{a \cos \theta} \mathcal{F}_{\theta} \\
\mathcal{F}_{\theta} M_{b} & =e^{i \pi b^{2} \sin \theta \cos \theta} T_{b \sin \theta} M_{b \cos \theta} \mathcal{F}_{\theta}=e^{-i \pi b^{2} \sin \theta \cos \theta} M_{b \cos \theta} T_{b \sin \theta} \mathcal{F}_{\theta} .
\end{aligned}
$$

Proof. Let $0<|\theta|<\pi$. Then;

$$
\begin{aligned}
\mathcal{F} & \left(T_{a} f\right)(\xi)=A_{\theta} \int_{\mathbb{R}} f(t-a) e^{\frac{-\pi i\left(2 t \xi+\left(\xi^{2}+t^{2}\right) \cos \theta\right)}{\sin \theta}} d t=A_{\theta} \int_{\mathbb{R}} f(u) e^{\frac{-\pi i\left(2(u+a) \xi+\left(\xi^{2}+(u+a)^{2}\right) \cos \theta\right)}{\sin \theta}} d u \\
& =A_{\theta} e^{\frac{-2 \pi i a \xi+\pi i \cos \theta\left(a^{2}+2 \xi a \cos \theta-a^{2} \cos ^{2} \theta\right)}{\sin \theta}} \int_{\mathbb{R}} f(u) e^{\frac{-2 \pi i u(\xi-a \cos \theta)}{\sin \theta}} e^{\frac{i \pi\left((\xi-a \cos \theta)^{2}+u^{2}\right) \cos \theta}{\sin \theta}} d u \\
& =e^{\pi i a^{2} \cos \theta \sin \theta} e^{-2 \pi i \xi a \sin \theta} f_{\theta}(\xi-a \cos \theta)=e^{-\pi i a^{2} \cos \theta \sin \theta} T_{a \cos \theta} M_{-a \sin \theta} f_{\theta}(\xi) .
\end{aligned}
$$


A similar calculation can be used to verify (3).

By combining (2) and (3) one can show that;

$$
\begin{aligned}
& \mathcal{F}_{\theta} T_{a} M_{b}=e^{-i \phi} e^{-2 \pi i a b \sin ^{2} \theta} T_{\alpha} M_{\beta} \mathcal{F}_{\theta}=e^{i \phi} e^{-2 \pi i a b \cos ^{2} \theta} M_{\beta} T_{\alpha} \mathcal{F}_{\theta} \\
& \mathcal{F}_{\theta} M_{b} T_{a}=e^{-i \phi} e^{2 \pi i a b \cos ^{2} \theta} T_{\alpha} M_{\beta} \mathcal{F}_{\theta}=e^{i \phi} e^{2 \pi i a b \sin ^{2} \theta} M_{\beta} T_{\alpha} \mathcal{F}_{\theta}
\end{aligned}
$$

where $\phi=\pi\left(a^{2}-b^{2}\right) \sin \theta \cos \theta$ and;

$$
\left[\begin{array}{c}
\alpha \\
\beta
\end{array}\right]=\left[\begin{array}{cc}
\cos \theta & \sin \theta \\
-\sin \theta & \cos \theta
\end{array}\right]\left[\begin{array}{l}
a \\
b
\end{array}\right]=\mathbf{R}_{-\theta}\left[\begin{array}{l}
a \\
b
\end{array}\right] .
$$

Lemma 1 shows that the fractional Fourier-transform can be associated with a rotation of the time-frequency plane. It turns out that $\mathcal{F}_{\theta}$ can be associated with a rotation of the complex plane in the Bargmann-Fock space as well. The Bargmann-Fock space $\mathcal{F}^{2}(\mathbb{C})$ is defined as;

$$
\mathcal{F}^{2}(\mathbb{C})=\left\{F \text { entire on } \mathbb{C}: \int_{\mathbb{C}}|F(z)|^{2} e^{-\pi|z|^{2}} d z<\infty\right\} .
$$

The Bargmann-transform $\mathcal{B}: L^{2}(\mathbb{R}) \rightarrow \mathcal{F}^{2}(\mathbb{C})$ is defined as;

$$
\mathcal{B} f(z)=2^{1 / 4} \int_{\mathbb{R}} f(t) e^{-\pi z^{2} / 2} e^{-\pi t^{2}} e^{2 \pi t z} d t, \quad z \in \mathbb{C} .
$$

The Bargmann-transform is a unitary mapping between $L^{2}(\mathbb{R})$ and $\mathcal{F}^{2}(\mathbb{C})$. For additional background about the Bargmann transform and the Bargmann-Fock space the author refers to e.g. [5, Ch. 3].

It is well-known that the Bargmann-transform treats the Fourier-transform as a $-\frac{\pi}{2}$-rotation of the complex plane. In other words; $\mathcal{B} \hat{f}(z)=R_{-\frac{\pi}{2}} \mathcal{B} f(z)$ where $R_{\theta} f(z)=f\left(z e^{i \theta}\right)$ denotes a rotation on $\mathbb{C}$. The following result shows that the fractional Fourier-transform shares a similar correspondence with $\mathcal{B}$.

Lemma 2. Let $0<|\theta|<\pi$. Then;

$$
\mathcal{B F}_{\theta}=R_{-\theta} \mathcal{B} .
$$

Proof. Consider $f \in L^{2}(\mathbb{R})$, and let $\omega^{2}=\frac{i \cos \theta}{\sin \theta}-1$. A direct calculation shows;

$$
\begin{aligned}
\mathcal{B} f_{\theta}(z) & =A_{\theta} e^{-\frac{\pi z^{2}}{2}\left(1+2 / \omega^{2}\right)} 2^{1 / 4} \int_{\mathbb{R}} f(\tau) e^{\pi \tau^{2}\left(\omega^{2}+1\right)} \int e^{\frac{-2 \pi i t \tau}{\sin \theta}} e^{\pi(t \omega+z / \omega)^{2}} d t d \tau \\
& =A_{\theta} e^{-\frac{\pi\left(z e^{-i \theta}\right)^{2}}{2}} 2^{1 / 4} \int_{\mathbb{R}} f(\tau) e^{-\pi \tau^{2}} e^{2 \pi \tau\left(z e^{-i \theta}\right)} A_{\theta}^{-1} d \tau=R_{-\theta} \mathcal{B} f(z) .
\end{aligned}
$$


As a consequence one can represent the fractional Fourier-transform as a composition of unitary operators on the Hilbert-spaces $L^{2}(\mathbb{R})$ and $\mathcal{F}^{2}(\mathbb{R})$, and is therefore unitary itself.

Corollary 1. The fractional Fourier-transform is unitary on $L^{2}(\mathbb{R})$.

From Lemma 2 it is clear that $\mathcal{F}_{\theta_{1}} \mathcal{F}_{\theta_{2}}=\mathcal{B}^{-1} R_{-\left(\theta_{1}+\theta_{2}\right)} \mathcal{B}=\mathcal{F}_{\theta_{1}+\theta_{2}}$. Thus the inverse fractional Fourier-transform is given as $\mathcal{F}_{\theta}^{-1}=\mathcal{F}_{-\theta}$. Using $\mathcal{F}_{\theta}$ and $\mathcal{F}_{-\theta}=\mathcal{F}_{\theta}^{-1}$ one can define other fractional operators such as fractional derivation and fractional convolution. The latter is defined as follows;

Definition 2 (Fractional convolution, [15]). Let $f, g \in L^{1} \cap L^{2}(\mathbb{R})$. The fractional convolution $f *_{\theta} g$ is defined as;

$$
f *_{\theta} g=\left(f_{-\theta} g_{-\theta}\right)_{\theta} .
$$

Note that $*_{0}$ and $*_{\pi / 2}=*$ correspond to usual multiplication and convolution.

Since the fractional Fourier-transform is unitary on $L^{2}(\mathbb{R})$ it follows that the Gabor system $\mathcal{G}\left(g, \mathbf{A} \mathbb{Z}^{2}\right)$ is a Gabor frame if and only if $\mathcal{G}\left(g_{\theta}, \mathbf{R}_{-\theta} \mathbf{A} \mathbb{Z}^{2}\right)$ is a frame;

$$
\begin{aligned}
A\left\|\mathcal{F}_{\theta} f\right\|_{2}^{2}=A\|f\|_{2}^{2} \leq & \sum_{\lambda \in \mathbf{A} \mathbb{Z}^{2}}\left|\left\langle f, \pi_{\lambda} g\right\rangle\right|^{2} \leq B\|f\|_{2}^{2}=B\left\|\mathcal{F}_{\theta} f\right\|_{2}^{2} \\
& \sum_{\lambda \in \mathbf{A} \mathbb{Z}^{2}}\left|\left\langle\mathcal{F}_{\theta} f, \mathcal{F}_{\theta} \pi_{\lambda} g\right\rangle\right|^{2}=\sum_{\mu \in \mathbf{R}_{-\theta} \mathbf{A} \mathbb{Z}^{2}}\left|\left\langle\mathcal{F}_{\theta} f, \pi_{\mu} \mathcal{F}_{\theta} g\right\rangle\right|^{2} .
\end{aligned}
$$

Similarly, if the Gabor frame operator $S_{g, \mathbf{A} \mathbb{Z}^{2}}$ associated with the Gabor system $\mathcal{G}\left(g, \mathbf{A} \mathbb{Z}^{2}\right)$ is bounded it follows that;

$$
\begin{aligned}
\mathcal{F}_{\theta}\left(S_{g, g, \mathbf{A} \mathbb{Z}^{2}} f\right) & =\sum_{\lambda \in \mathbf{A} \mathbb{Z}^{2}}\left\langle f, \pi_{\lambda} g\right\rangle \mathcal{F}_{\theta} \pi_{\lambda} g=\sum_{\mu \in \mathbf{R}_{-\theta} \mathbf{A} \mathbb{Z}^{2}}\left\langle\mathcal{F}_{\theta} f, \pi_{\mu} \mathcal{F}_{\theta} g\right\rangle \pi_{\mu} \mathcal{F}_{\theta} g \\
& =S_{g_{\theta}, g_{\theta}, \mathbf{R}_{-\theta} \mathbf{A} \mathbb{Z}^{2} f_{\theta} .}
\end{aligned}
$$

In particular, the fractional Fourier-transform rotates the lattice associated with the Gabor frame operator.

\section{Representations of the Gabor frame operator for non-rectangular lattices.}

The Walnut representation theorem is one of the basic results regarding the Gabor frame operator corresponding to rectangular lattices $\Lambda=a \mathbb{Z} \times b \mathbb{Z}$. It 
represents the (two windows) Gabor frame operator $S_{g, \gamma, \Lambda}=\sum\left\langle f, \pi_{\lambda} g\right\rangle \pi_{\lambda} \gamma$ on the form;

$$
S_{g, \gamma, \Lambda} f(t)=b^{-1} \sum_{n} G_{n}(t) T_{\frac{n}{b}} f(t)
$$

where $G_{n}(t)=\sum_{k} T_{a k} \gamma(t) T_{a k+\frac{n}{b}} \bar{g}(t)$.

The representation (5) and the correlation function $G_{n}(t)$ can be used to prove that the Gabor frame operator is bounded on $L^{2}(\mathbb{R})$ if the windows $g, \gamma$ belong to the Wiener-space, [5]. The Wiener space can be defined as;

$$
W(\mathbb{R})=\left\{f \in L^{\infty}(\mathbb{R}): \sum_{k}\left\|\chi_{[k, k+1[} f\right\|_{\infty}<\infty\right\}
$$

with norm given by;

$$
\|f\|_{W}=\sum_{k}\left\|\chi_{[k, k+1[} f\right\|_{\infty}
$$

In this section we consider generalizations of the Walnut representation theorem to non-rectangular lattices in $\mathbb{R}^{2}$.

We first introduce a Walnut representation formula for the Gabor frame operator corresponding to vertically sheared lattices, i.e. lattices in the form $\mathbf{S} \Lambda=\{(a k, s b k+b n): k, n \in \mathbb{Z}\}$. Note that for such lattices the time component is independent of shifts in frequency.

Lemma 3 (Walnut representation theorem for vertically sheared lattices). Let $g, \gamma \in W(\mathbb{R})$ and consider the lattice $\mathbf{S} \Lambda=\{(a k, s b k+b n): k, n \in \mathbb{Z}\}$ for some $0 \leq s<1$. The Gabor frame operator can be represented as;

$$
\begin{aligned}
S_{g, \gamma, \mathbf{S} \Lambda} f(t) & =\frac{1}{b} \sum_{n}\left(\sum_{k} \gamma(t-a k) \overline{g\left(t-a k-\frac{n}{b}\right)} e^{2 \pi i s n k}\right) T_{\frac{n}{b}} f(t) \\
& =\frac{1}{b} \sum_{n} G_{n}(t) T_{\frac{n}{b}} f(t) .
\end{aligned}
$$

The Gabor frame operator is bounded on $L^{2}(\mathbb{R})$ with norm;

$$
\left\|S_{g, \gamma, \mathbf{S} \Lambda} f\right\|_{2} \leq 2\left(1+a^{-1}\right)\left(1+b^{-1}\right)\|g\|_{W}\|\gamma\|_{W}\|f\|_{2} .
$$

Proof. A detailed proof of the Lemma follows the pattern of the proof in [5, Th. 6.3.2]. The following calculations sketch how one obtains (6) and (7).

Let us first verify $(6)$. Let $f \in L^{2}(\mathbb{R})$ and consider the lattice $\mathbf{S} \Lambda=\{(a k, s b k+b n)$ : $k, n \in \mathbb{Z}\}$. The Gabor frame operator can be written as;

$$
S_{g, \gamma, \Lambda} f(t)=\sum_{k} M_{s b k} T_{a k} \gamma(t) \sum_{n}\left\langle f M_{-s b k} T_{a k} \bar{g}, M_{b n}\right\rangle M_{b n} .
$$


The inner sum has the form;

$$
\sum_{n}\left\langle f M_{-s b k} T_{a k} \bar{g}, M_{b n}\right\rangle M_{b n}=\sum_{n} \hat{h}(b n) e^{2 \pi i b n t},
$$

where $h(\tau)=f(\tau) M_{-s b k} T_{a k} \bar{g}(\tau)$. Note that $h \in L^{2}(\mathbb{R})$ since $g \in W(\mathbb{R})$ is bounded. Now the Poisson summation formula gives;

$$
S_{g, \gamma, \Lambda} f(x)=\frac{1}{b} \sum_{k} M_{s b k} T_{a k} \gamma(t) \sum_{n} T_{\frac{n}{b}} f(t) T_{\frac{n}{b}+a k} \bar{g}(t) e^{-2 \pi i\left(t-\frac{n}{b}\right) s b k} .
$$

The representation (6) follows by rearranging the order of summation.

Since $g, \gamma \in W(\mathbb{R})$ the functions are bounded and it follows that the correlation function $G_{n}(t)=\sum_{k} \gamma(t-a k) \overline{g\left(t-a k-\frac{n}{b}\right)} e^{2 \pi i s n k}$ is bounded and;

$$
\left\|G_{n}\right\|_{\infty}=\sup _{t}\left|\sum_{k} \gamma(t-a k) \bar{g}\left(t-a k-\frac{n}{b}\right) e^{2 \pi i s n k}\right| \leq \sup _{t} \sum_{k}\left|\gamma(t-a k) \overline{g\left(t-a k-\frac{n}{b}\right)}\right| .
$$

The operator bound (7) follows from similar calculations as in [5, L. 6.3.1].

The previous lemma gives an analog of the Walnut representation theorem for vertically sheared lattices. The modified representation differs from the original by a modulation term in the correlation-function $G_{n}$. This term corresponds to the shearing of the lattice $\mathbf{S} \Lambda$.

Let now $\mathbf{A} \mathbb{Z}^{2}$ denote a lattice in $\mathbb{R}^{2}$. There exist a $s \in[0,1)$, a $\theta \in(-\pi, \pi)$, and a rectangular lattice $\Lambda=a \mathbb{Z} \times b \mathbb{Z}$ such that $\mathbf{A} \mathbb{Z}^{2}$ can be represented in the form $\mathbf{R}_{\theta} \mathbf{S} \Lambda$. By (4) it follows that $\mathcal{F}_{\theta}\left(S_{g, \gamma, \mathbf{R}_{\theta} \mathbf{S} \Lambda} f\right)=S_{g_{\theta}, \gamma_{\theta}, \mathbf{S} \Lambda} f_{\theta}$. Consequently, the Gabor frame operator associated to a lattice of the form $\mathbf{R}_{\theta} \mathbf{S} \Lambda$ can be transformed into a Gabor frame operator associated to some lattice $\mathbf{S} \Lambda$.

Motivated by these observations we consider a modified Walnut representation of the Gabor frame operator $S_{g, \gamma, \mathbf{R}_{\theta} \mathbf{S} \Lambda} f$.

Theorem 1 (Modified Walnut representation theorem). Let $g, \gamma \in W \cap \mathcal{F}_{\theta} W(\mathbb{R})$ and consider the lattice $\mathbf{R}_{\theta} \mathbf{S} \Lambda$ for some $|\theta|<\pi$ and $0 \leq s<1$. The Gabor frame operator $S_{g, \gamma, \mathbf{R}_{\theta} \mathbf{S} \Lambda} f$ can be represented as;

$$
\mathcal{F}_{\theta} S_{g, \gamma, \mathbf{R}_{\theta} \mathbf{S} \Lambda} f(t)=\frac{1}{b} \sum_{n} G_{n}(t) T_{\frac{n}{b}} f_{\theta}(t)
$$

where

$$
G_{n}(t)=\sum_{k} \gamma_{\theta}(t-a k) \overline{g_{\theta}\left(t-a k-\frac{n}{b}\right)} e^{2 \pi i s n k} .
$$

The Gabor frame operator $S_{g, \gamma, \mathbf{R}_{\theta} \mathbf{S} \Lambda}$ is a bounded on $L^{2}(\mathbb{R})$ with norm;

$$
\left\|S_{g, \gamma, \Lambda} f\right\|_{2} \leq 2\left(1+a^{-1}\right)\left(1+b^{-1}\right)\left\|g_{\theta}\right\|_{W}\left\|\gamma_{\theta}\right\|_{W}\|f\|_{2} .
$$


Proof. Let $f \in L^{2}(\mathbb{R})$. By unitarity of the fractional Fourier-transform and (7) it is clear that $S_{g, \gamma, \mathbf{R}_{\theta} \mathbf{S} \Lambda}$ is bounded on $L^{2}(\mathbb{R})$ with norm;

$$
\left\|S_{g, \gamma, \mathbf{R}_{\theta} \mathbf{S} \Lambda} f\right\|_{2}=\left\|S_{g_{\theta}, \gamma_{\theta}, \mathbf{S} \Lambda} f_{\theta}\right\|_{2} \leq 2\left(1+a^{-1}\right)\left(1+b^{-1}\right)\left\|g_{\theta}\right\|_{W}\left\|\gamma_{\theta}\right\|_{W}\left\|f_{\theta}\right\|_{2} .
$$

By (4) and Lemma 3 one obtains $\mathcal{F}_{\theta} S_{g, \gamma, \mathbf{R}_{\theta} \mathbf{S} \Lambda} f=S_{g_{\theta}, \gamma_{\theta}, \mathbf{S} \Lambda} f_{\theta}=\frac{1}{b} \sum_{n} G_{n} T_{\frac{n}{b}} f_{\theta}$ where $G_{n}$ is given by (9).

Note that by taking the inverse fractional Fourier-transform on both sides of (8) it follows that;

$$
S_{g, \gamma, \mathbf{R}_{\theta} \mathbf{S} \Lambda} f(t)=\frac{1}{b} \sum_{n} \mathcal{F}_{-\theta}\left(G_{n} T_{\frac{n}{b}} f_{\theta}\right)(t)=\frac{1}{b} \sum_{n} \mathcal{F}_{-\theta}\left(G_{n}\right) *_{-\theta} \tilde{f}(t),
$$

where $\tilde{f}(t)=e^{\frac{i}{2}\left(\frac{n}{b}\right)^{2} \sin \theta \cos \theta} T_{\frac{n}{b} \cos \theta} M_{\frac{n}{b}} \sin \theta f(t)$. The last equality holds in sense of distributions.

Intuitively one can interpret the original Walnut-representation and (6) as a correspondence between the Gabor frame operator and the time-concentration of the signal $f$. Similarly, one can interpret (8) as a correspondence between the mixed time-frequency content of the Gabor frame operator and that of the signal. The mutual weighting between the time and frequency depends on the shape of the fundamental domain of the lattice.

\section{Diagonalization of the Gabor frame operator at critical density.}

Let $\Lambda=a \mathbb{Z} \times b \mathbb{Z}, a b=1$ be a rectangular lattice at critical density. The classical Zak-transform;

$$
\mathcal{Z}_{\alpha} f(t, \omega)=\sum_{n} f(t-\alpha n) e^{2 \pi i \alpha n \omega}
$$

diagonalizes the Gabor frame operator $S_{g, \gamma, \Lambda} f(t)$ corresponding to rectangular lattices at critical density, see e.g. [5, Ch. 8]. The diagonalization is of the form;

$$
\mathcal{Z}_{a}\left(S_{g, \gamma, \Lambda} f\right)=a \overline{\mathcal{Z}_{a} g} \mathcal{Z}_{a} \gamma \mathcal{Z}_{a} f
$$

The previous identity holds in the case of critical oversampling $a b=1$. In the case of rational oversampling $a b=\frac{p}{q}<1$ the Zibulski-Zeevi representation uses Zak-transform methods to represent the Gabor frame operator in the form $\overrightarrow{\mathcal{Z}}_{a} S_{g, \gamma, \Lambda} f=\mathbf{Z} \overrightarrow{\mathcal{Z}}_{a} f,[21]$. In this case $\overrightarrow{\mathcal{Z}}_{a} S_{g, \gamma, \Lambda} f$ and $\overrightarrow{\mathcal{Z}}_{a} f$ are vectors of length $p$, and $\mathbf{Z}$ is a $p \times p$-matrix. As a consequence the Gabor system $\mathcal{G}(g, \Lambda)$ is a Gabor frame if and only if $\left|\mathcal{Z}_{a} g(t, \omega)\right|$ is non-zero and bounded - or in the vector case if and only if the eigenvalues of $\mathbf{Z}$ are non-zero and bounded. 
In this section we construct a 'modified' Zak-transform which diagonalizes the Gabor frame operator corresponding to any lattice $\mathbf{A} \mathbb{Z}^{2}=\mathbf{R}_{\theta} \mathbf{S} \Lambda$ at critical density in $\mathbb{R}^{2}$.

The 'modified' Zak-transform is the sum of $\left\{f_{\theta}(t-\alpha n) e^{-\pi i s n^{2}} e^{2 \pi i \alpha \omega n}\right\}_{n}$.

Definition 3 (Modified Zak-transform). Let $0 \leq s<1$ and $0 \leq|\theta|<\pi$ be given. The modified Zak-transform is defined as;

$$
\mathcal{Z}_{\alpha, s, \theta} f(t, \omega)=\sum_{n} f_{\theta}(t-\alpha n) e^{-\pi i s n^{2}} e^{2 \pi i \alpha \omega n} .
$$

Observe that for $s=0$ and $\theta=0$ the modified Zak-transform coincides with the original one. In what follows we denote $\mathcal{Z}_{\alpha, s}=\mathcal{Z}_{\alpha, s, 0}$. Note that for the quincunx-lattice $\{(a k, s b k+b n): k, n \in \mathbb{Z}\}$ a similar modified Zak-transform was discovered by M. Bastiaans and A. Leest in [2].

The modified Zak-transform shares most of the properties with the 'original' one. For a survey of the Zak-transform see e.g. [7]. The modified Zak-transform $\mathcal{Z}_{\alpha, s, \theta} f(t, \omega)$ is quasi-periodic on the fundamental domain $\mathbf{S}([0, \alpha) \times[0,1 / \alpha))$, i.e;

$$
\begin{gathered}
\mathcal{Z}_{\alpha, s, \theta} f\left(x, \omega+\frac{k}{\alpha}\right)=\mathcal{Z}_{\alpha, s, \theta} f(x, \omega) \\
\mathcal{Z}_{\alpha, s, \theta} f\left(t+k \alpha, \omega+\frac{s k}{\alpha}\right)=e^{\pi i s k^{2}} e^{2 \pi i \alpha \omega k} \mathcal{Z}_{\alpha, s, \theta} f(t, \omega) .
\end{gathered}
$$

The value of the modified Zak-transform is therefore completely determined by its value on the fundamental domain.

The inverse transform is defined as;

$$
\mathcal{Z}_{\alpha, s, \theta}^{-1} F(t)=\mathcal{F}_{-\theta}\left(\int_{0}^{\frac{1}{\alpha}} F(t, \omega) d \omega\right)
$$

for $F \in L^{2}\left(\mathbf{S}\left([0, \alpha) \times\left[0, \frac{1}{\alpha}\right)\right)\right)$. In the Appendix we show that $\alpha^{1 / 2} \mathcal{Z}_{\alpha, s, \theta}$ is unitary considered as an operator between $L^{2}(\mathbb{R})$ and $L^{2}(\mathbf{S}([0, \alpha) \times[0,1 / \alpha)))$.

The translation and modulation operators commute with respect to rectangular lattices at critical density, i.e. $T_{a k} M_{\frac{n}{a}}=M_{\frac{n}{a}} T_{a k}$ for all $n, k \in \mathbb{Z}$. Consequently there exists a unitary operator which simultaneously diagonalizes the translation and modulation operators, [3]. It is well-known that the (unitary) Zak-transform diagonalizes time-frequency shifts and the Gabor frame operator corresponding to lattices on the form $a \mathbb{Z} \times a^{-1} \mathbb{Z}$.

In contrast to the rectangular case, the translation- and modulation-operator do not commute with respect to non-rectangular lattices. Consider instead the joint translation- and modulation-operators $\mathcal{T}=T_{a k} M_{s b k+b n}$ and $\mathcal{S}=M_{s b k+b n} T_{a k}$ associated to the lattice $\mathbf{S} \Lambda$ at critical density. Both $\mathcal{S}$ and $\mathcal{T}$ commute with their respective adjoints and are therefore normal operators on $L^{2}(\mathbb{R})$. By the spectral theorem it follows that there exists a measure space $(X, \mu)$, functions 
$M_{\mathcal{S}}, M_{\mathcal{T}} \in L^{\infty}(X, \mu)$ and unitary operators $U, V: L^{2}(\mathbb{R}) \rightarrow L^{2}(X, \mu)$ which diagonalizes respectively $\mathcal{S}$ and $\mathcal{T}[13$, Ch. 6]. One can show that $\mathcal{S} \mathcal{T}=\mathcal{T} \mathcal{S}$, hence $\mathcal{S}$ and $\mathcal{T}$ can be diagonalized simultaneously. It follows that $U=V$ and that the operators $\mathcal{S}$ and $\mathcal{T}$ can be represented in the form;

$$
\mathcal{S}=U^{*} M_{\mathcal{S}} U, \quad \mathcal{T}=U^{*} M_{\mathcal{T}} U .
$$

These abstract considerations can be realized by using the modified Zaktransform $\mathcal{Z}_{\alpha, s, \theta}: L^{2}(\mathbb{R}) \rightarrow L^{2}(\mathbf{S}([0, \alpha) \times[0,1 / \alpha)))$. We show that the modified Zak-transform $\mathcal{Z}_{a, s}$ diagonalizes the joint time-frequency shift operators $\mathcal{S}$ and $\mathcal{T}$ associated with lattices in the form $\mathbf{S} \Lambda$ at critical density.

Lemma 4 (Diagonalization of $\mathcal{S}$ and $\mathcal{T}$ ). Let $\mathcal{T}=T_{a k} M_{s b k+b n}$ and $\mathcal{S}=M_{s b k+b n} T_{a k}$ with $a b=1$ and $k, n \in \mathbb{Z}$. The modified Zak-transform satisfies;

$$
\begin{aligned}
& \mathcal{Z}_{a, s} \mathcal{T} f(t, \omega)=M_{\mathcal{T}}(t, \omega) \mathcal{Z}_{a, s} f(t, \omega) \\
& \mathcal{Z}_{a, s} \mathcal{S} f(t, \omega)=M_{\mathcal{S}}(t, \omega) \mathcal{Z}_{a, s} f(t, \omega)
\end{aligned}
$$

where $M_{\mathcal{T}}(t, \omega)=e^{2 \pi i s k b t} e^{-\pi i s k^{2}} e^{2 \pi i b n t} e^{-2 \pi i a \omega k}$ and $M_{\mathcal{S}}(t, \omega)=\overline{M_{\mathcal{T}}(-t,-\omega)}$.

Proof. By inserting $\mathcal{S} f$ into the definition of $\mathcal{Z}_{a, s}$ it follows that;

$$
\begin{aligned}
\mathcal{Z}_{a, s} \mathcal{S} f(t, \omega) & =\sum_{j} f(t-a(j+k)) e^{-\pi i s j^{2}} e^{2 \pi i(s b k+b n)(t-a j)} e^{2 \pi i a \omega j} \\
& =e^{2 \pi i(s b k+b n) t} \mathcal{Z}_{a, s} f(t-a k, \omega-s b k-b n)
\end{aligned}
$$

By quasi-periodicity one obtains;

$$
\mathcal{Z}_{a, s} \mathcal{S} f(t, \omega)=e^{2 \pi i(s b k+b n) t} e^{\pi i s k^{2}} e^{-2 \pi i a \omega k} \mathcal{Z}_{a, s} f(t, \omega) .
$$

The proof of (10) is similar.

Note that the lemma can be generalized to lattices on the form $\mathbf{R}_{\theta} \mathbf{S} \Lambda$ by using $\mathcal{Z}_{a, s, \theta}$ and Lemma 1.

The next lemma proves that the suggested Zak-transform $\mathcal{Z}_{a, s}$ diagonalizes the Gabor frame operator corresponding to lattices on the form $\mathbf{S} \Lambda$.

Lemma 5. Let $g, \gamma \in W(\mathbb{R})$. Consider the lattice $\mathbf{S} \Lambda$ for some $0 \leq s<1$ and $a b=1$. The modified Zak-transform diagonalizes $S_{g, \gamma, \mathbf{S} \Lambda} f$ for $f \in L^{2}(\mathbb{R})$, i.e;

$$
\mathcal{Z}_{a, s}\left(S_{g, \gamma, \mathbf{S} \Lambda} f\right)=a \overline{\mathcal{Z}_{a, s} g} \mathcal{Z}_{a, s} \gamma \mathcal{Z}_{a, s} f
$$

Proof. To prove this result we use the corresponding modified Walnut representation (6). 
For any $h \in L^{2}(\mathbb{R})$ it follows that;

$$
\begin{aligned}
& \left\langle S_{g, \gamma, \mathbf{S} \Lambda} f, h\right\rangle_{L^{2}(\mathbb{R})}=b^{-1} \int_{\mathbb{R}} \sum_{n} G_{n}(t) f\left(t-\frac{n}{b}\right) \overline{h(t)} d t \\
& =b^{-1} \sum_{j} \int_{0}^{1 / b} \sum_{n} G_{n}\left(t-\frac{j}{b}\right) f\left(t-\frac{n+j}{b}\right) \overline{h\left(t-\frac{j}{b}\right)} d t \\
& =b^{-1} \int_{0}^{1 / b} \sum_{j, n}\left(\sum_{k} \gamma\left(t-\frac{j}{b}-a k\right) \overline{g\left(t-\frac{j}{b}-a k-\frac{n}{b}\right)} e^{2 \pi i s n k}\right) f\left(t-\frac{n+j}{b}\right) \overline{h\left(t-\frac{j}{b}\right)} d t
\end{aligned}
$$

Using $a b=1$ and the substitutions $l=n+j, r=k+j$ and $m=l-j$ successively one can write $\left\langle S_{g, \gamma, \mathbf{S} \Lambda} f, h\right\rangle_{L^{2}(\mathbb{R})}$ as;

$$
a \int_{0}^{a} \sum_{j}\left(\sum_{m} \sum_{r} \gamma(t-a r) \overline{g(t-a r-a m)} e^{2 \pi i s(r-j) m}\right) f\left(t-\frac{m+j}{b} \overline{h\left(t-\frac{j}{b}\right)} d t .\right.
$$

The integrand has the form of an $\ell_{2}$ inner-product $\langle a, b\rangle_{\ell^{2}(\mathbb{Z})}$ where $a=\left\{\sum_{m, r} \gamma(t-a r) \overline{g(t-a r-a m)} e^{2 \pi i s(r-j) m} f\left(t-\frac{m+j}{b}\right)\right\}_{j}$ and $b=\left\{h\left(t-\frac{j}{b}\right)\right\}_{j}$. By Plancherel's formula and a coordinate-wise multiplication with a Gaussian chirp one obtains;

$$
\begin{aligned}
\langle a, b\rangle_{\ell^{2}(\mathbb{Z})} & =\left\langle a e^{-\pi i s(\cdot)^{2}}, b e^{-\pi i s(\cdot)^{2}}\right\rangle_{\ell^{2}(\mathbb{Z})}=\left\langle\sum_{j} a_{j} e^{-\pi i s j^{2}} e^{2 \pi i a \omega j}, \sum_{j} b_{j} e^{-\pi i s j^{2}} e^{2 \pi i a \omega j}\right\rangle_{L^{2}\left(\left[0, \frac{1}{a}\right)\right)} \\
& =\left\langle\sum_{j} a_{j} e^{-\pi i s j^{2}} e^{2 \pi i a \omega j}, \mathcal{Z}_{a, s} h(t, \omega)\right\rangle_{L^{2}\left(\left[0, \frac{1}{a}\right)\right)} .
\end{aligned}
$$

By studying the series $\sum_{j} a_{j} e^{-\pi i a s j^{2}}$ one can calculate;

$$
\begin{aligned}
& \sum_{j} a_{j} e^{-\pi i s j^{2}} e^{2 \pi i a \omega j}=\sum_{m, r} \gamma(t-a r) \overline{g(t-a r-a m)} e^{2 \pi i s r m} e^{-2 \pi i a m \omega} e^{\pi i s m^{2}} \\
& \sum_{j} f(t-a(m+j)) e^{-\pi i s(m+j)^{2}} e^{2 \pi i a(j+m) \omega} \\
& =\mathcal{Z}_{a, s} f(t, \omega) \sum_{r} \gamma(t-a r) e^{-\pi i s r^{2}} e^{2 \pi i a r \omega} \sum_{m} \overline{g(t-a(m+r))} e^{\pi i s(m+r)^{2}} e^{-2 \pi i a(m+r) \omega} \\
& =\overline{\mathcal{Z}_{a, s} g}(t, \omega) \mathcal{Z}_{a, s} \gamma(t, \omega) \mathcal{Z}_{a, s} f(t, \omega) .
\end{aligned}
$$

In other words, for arbitrary $h \in L^{2}(\mathbb{R})$ we have;

$$
\left\langle\mathcal{Z}_{a, s} S_{g, \gamma, \mathbf{S} \Lambda} f, \mathcal{Z}_{a, s} h\right\rangle_{L^{2}\left([0, a) \times\left[0, \frac{1}{a}\right)\right)}=\left\langle a \overline{\mathcal{Z}_{a, s} g} \mathcal{Z}_{a, s} \gamma \mathcal{Z}_{a, s} f, \mathcal{Z}_{a, s} h\right\rangle_{L^{2}\left([0, a) \times\left[0, \frac{1}{a}\right)\right)} .
$$

Therefore;

$$
\mathcal{Z}_{a, s}\left(S_{g, \gamma, \mathbf{S} \Lambda} f\right)=a \overline{\mathcal{Z}_{a, s} g(t, \omega)} \mathcal{Z}_{a, s} \gamma(t, \omega) \mathcal{Z}_{a, s} f(t, \omega)
$$


The general result follows by the definition of $\mathcal{Z}_{a, s, \theta}$ and unitarity of the fractional Fourier-transform.

Theorem 2. Let $g, \gamma \in W \cap \mathcal{F}_{\theta} W(\mathbb{R})$. Consider the lattice $\mathbf{R}_{\theta} \mathbf{S} \Lambda$ for some $\theta \in(-\pi, \pi), 0 \leq s<1$ and $a b=1$. The modified Zak-transform diagonalizes $S_{g, \gamma, \mathbf{R}_{\theta} \mathbf{S} \Lambda} f$ for $f \in L^{2}(\mathbb{R})$, i.e;

$$
\mathcal{Z}_{a, s, \theta}\left(S_{g, \gamma, \mathbf{R}_{\theta} \mathbf{S} \Lambda} f\right)=a \mathcal{Z}_{a, s, \theta} \gamma \overline{\mathcal{Z}_{a, s, \theta} g} \mathcal{Z}_{a, s, \theta} f
$$

Proof. By using (4) and Lemma 5 it follows that;

$$
\begin{aligned}
\mathcal{Z}_{a, s, \theta}\left(S_{g, \gamma, \mathbf{R}_{\theta} \mathbf{S} \Lambda} f\right) & =\mathcal{Z}_{a, s}\left(S_{g_{\theta}, \gamma_{\theta}, \mathbf{S} \Lambda} f_{\theta}\right)=a \mathcal{Z}_{a, s}\left(\mathcal{F}_{\theta} \gamma\right) \overline{\mathcal{Z}_{a, s}\left(\mathcal{F}_{\theta} g\right)} \mathcal{Z}_{a, s}\left(\mathcal{F}_{\theta} f\right) \\
& =a \mathcal{Z}_{a, s, \theta} \gamma \overline{\mathcal{Z}_{a, s, \theta} g} \mathcal{Z}_{a, s, \theta} f .
\end{aligned}
$$

\section{Discussion}

In this article we have studied Gabor systems for non-rectangular lattices. We have presented a new Zak-transform which diagonalizes the Gabor frame operator corresponding to non-rectangular lattices at critical density. We have also suggested a representation of the Gabor frame operator similar to the classical Walnut representation. The study is based on properties of the fractional Fourier-transform.

The Walnut representation theorem and Zak-transform are central results about Gabor systems corresponding to rectangular lattices. The method presented in this article allows one to extend important results about the structure of the Gabor frame operator from the rectangular to the non-rectangular case. The modified Zak-transform gives immediate conditions on the windows ensuring that the Gabor system is a frame at critical density. Our study also shows a new approach to consider Gabor systems on non-rectangular lattices, different from the conventional methods based on the representation theory on the Heisenberg-group.

\section{ACKNOWLEDGEMENT}

The author would first of all like to thank his supervisor Prof. Yurii Lyubarskii for the support and guidance during the work.

The author would also like to thank Prof. Karlheinz Gröchenig for useful discussion. 
P. NES

\section{References}

[1] L. B. Almeida, The fractional Fourier transform and time-frequency representations, IEEE transactions on Signal Processing, 42(11), 3084-3091, 1994.

[2] M. J. Bastiaans and A. J. van Leest, Modified Zak transform for the quincunx-type Gabor lattice. In Proc. TFTSA-98, pages 173 -176, oct. 1998.

[3] J. B. Conway, A Course in Functional Analysis, Springer-Verlag, New York, 2nd edition, 1990.

[4] H. G. Feichtinger and K. Gröchenig, Gabor frames and time-frequency analysis of distributions, Journal of Functional Analysis, 146(2), $464-$ 495, 1997.

[5] K. Gröchenig, Foundations of Time-Frequency Analysis, Birkhäuser, Boston, 2001.

[6] C. Heil, History and evolution of the density theorem for Gabor frames, Journal of Fourier Analysis and Applications, 13, 113-166, 2007.

[7] A. J. E. M. Janssen, The Zak transform: A signal transform for sampled time-continuous signals, Philips J. Res., 43(1), 23-69, 1988.

[8] A. J. E. M. Janssen, Zak Transforms with few zeros and the tie. In Advances in Gabor Analysis, pages 31-70. Birkhäuser Boston, 2003.

[9] A. J. E. M. Janssen and T. Strohmer, Hyperbolic secants yield Gabor frames, Appl. Comput. Harmon. Anal., 12(2), 259-267, 2002.

[10] B.-Z. Li, R. Tao, T.-Z. Xu and Y. Wang, The Poisson sum formulae associated with the fractional Fourier transform, Signal Process., 89(5), 851-856, 2009.

[11] A. W. Lohmann, Image rotation, Wigner rotation, and the fractional Fourier transform, J. Opt. Soc. Am. A, 10(10), 2181-2186, 1993.

[12] A. W. Lohmann and D. Mendlovic, Fractional Fourier transform: Photonic implementation, Appl. Opt., 33(32), 7661-7664, 1994.

[13] B. D. MacCluer, Elementary functional analysis. Springer, 2008.

[14] A. C. McBride and F. H. Kerr. On Namias's fractional Fourier transforms. IMA Journal of Applied Mathematics, 39(2):159-175, 1987. 
[15] D. Mustard, Fractional convolution, J. Austral. Math. Soc. Ser. B, 40(2), 257-265, 1998.

[16] V. Namias, The fractional order Fourier transform and its application to quantum mechanics, IMA Journal of Applied Mathematics, 25(3), 241-265, 1980 .

[17] H. M. Ozaktas, Z. Zalevsky and M. A. Kutay, The Fractional Fourier Transform with Applications in Optics and Signal Processing, John Wiley and Sons, 2001.

[18] R. Saxena and K. Singh, Fractional Fourier transform: A novel tool for signal processing, J. Indian Inst. Sci., 85, $11-26,2005$.

[19] D. F. Walnut, Continuity properties of the Gabor frame operator, Journal of Mathematical Analysis and Applications, 165(2), 479 - 504, 1992.

[20] A. I. Zayed, Hilbert transform associated with the fractional Fourier transform, Signal Processing Letters, IEEE, 5(8), 206 -208, 1998.

[21] M. Zibulski and Y. Y. Zeevi, Analysis of multiwindow Gabor-type schemes by frame methods, Applied and Computational Harmonic Analysis, 4(2), $188-221,1997$.

\section{Appendix A: The Plancherel identity and unitarity of Zak-transform.}

The modified Zak-transform $\mathcal{Z}_{\alpha, s, \theta}$ is unitary considered as a mapping between $L^{2}(\mathbb{R})$ and $L^{2}\left(\mathbf{S}\left([0, \alpha) \times\left[0, \frac{1}{\alpha}\right)\right)\right)$. A proof of this statement follows same arguments as in the proof [5, Th. 8.2.3].

Lemma 6 (Plancherel's identity). Let $f \in L^{2}(\mathbb{R})$. Then;

$$
\iint_{\mathbf{S}\left([0, \alpha) \times\left[0, \frac{1}{\alpha}\right)\right)}\left|\mathcal{Z}_{\alpha, s, \theta} f(t, \omega)\right|^{2} d t d \omega=\frac{1}{\alpha}\|f\|_{2}^{2} .
$$

Proof. The modified Zak-transform can be interpreted as the Fourier-series of the sequence $\left\{f_{\theta}(t-\alpha n) e^{-\pi i s n^{2}}\right\}_{n \in \mathbb{Z}} \in \ell^{2}$. The Plancherel's theorem for Fourierseries gives that for almost every $t$;

$$
\int_{0}^{\frac{1}{\alpha}}\left|\mathcal{Z}_{\alpha, s, \theta} f(t, \omega)\right|^{2} d \omega=\frac{1}{\alpha} \sum_{n}\left|f_{\theta}(t-\alpha n) e^{-\pi i s n^{2}}\right|^{2} .
$$

Integrating this identity with respect to $t$ yields;

$$
\int_{0}^{\alpha} \int_{0}^{\frac{1}{\alpha}}\left|\mathcal{Z}_{\alpha, s, \theta} f(t, \omega)\right|^{2} d \omega d t=\frac{1}{\alpha} \sum_{n} \int_{0}^{\alpha}\left|f_{\theta}(t-\alpha n)\right|^{2} d t=\frac{1}{\alpha} \int_{\mathbb{R}}\left|f_{\theta}(t)\right|^{2} d t=\frac{1}{\alpha}\|f\|_{2}^{2} .
$$


By a change of variable one can show that;

$$
\int_{0}^{\alpha} \int_{0}^{\frac{1}{\alpha}}\left|\mathcal{Z}_{\alpha, s, \theta} f(t, \omega)\right|^{2} d \omega d t=\iint_{\mathbf{S}\left([0, \alpha) \times\left[0, \frac{1}{\alpha}\right)\right)}\left|\mathcal{Z}_{\alpha, s, \theta} f\left(\mathbf{S}^{-1}(t, \omega)\right)\right|^{2} d \omega d t .
$$

However, since the modified Zak-transform is $\frac{1}{\alpha}$-periodic with respect to $\omega$ it follows that;

$$
\iint_{\mathbf{S}\left([0, \alpha) \times\left[0, \frac{1}{\alpha}\right)\right)}\left|\mathcal{Z}_{\alpha, s, \theta} f\left(\mathbf{S}^{-1}(t, \omega)\right)\right|^{2} d \omega d t=\iint_{\mathbf{S}\left([0, \alpha) \times\left[0, \frac{1}{\alpha}\right)\right)}\left|\mathcal{Z}_{\alpha, s, \theta} f(t, \omega)\right|^{2} d \omega d t .
$$

Theorem 3 (Unitarity). The modified Zak-transform $\alpha^{1 / 2} \mathcal{Z}_{\alpha, s, \theta}$ is a unitary operator between $L^{2}(\mathbb{R})$ and $L^{2}\left(\mathbf{S}\left([0, \alpha) \times\left[0, \frac{1}{\alpha}\right)\right)\right)$.

Proof. We will first verify the theorem in the case $\theta=0$. By the previous lemma it follows that $\alpha^{1 / 2} \mathcal{Z}_{\alpha, s}: L^{2}(\mathbb{R}) \rightarrow L^{2}\left(\mathbf{S}\left([0, \alpha) \times\left[0, \frac{1}{\alpha}\right)\right)\right)$ and $\left\|\mathcal{Z}_{\alpha, s, \theta} f\right\|=\|f\|_{2}$. As in [5, Th. 8.2.3] it suffices to show that the modified Zak-transform maps an orthonormal basis in $L^{2}(\mathbb{R})$ to an orthonormal basis in $L^{2}\left(\mathbf{S}\left([0, \alpha) \times\left[0, \frac{1}{\alpha}\right)\right)\right)$.

Consider the orthonormal basis in $L^{2}(\mathbb{R})$ given by $\left\{M_{\frac{n}{\alpha}} T_{\alpha k} \chi_{[0, \alpha)}\right\}_{n, k}$, where $\chi$ denotes the usual indicator function. For almost every $(t, \omega) \in \mathbf{S}\left([0, \alpha) \times\left[0, \frac{1}{\alpha}\right)\right)$ one can show that;

$$
\begin{aligned}
\mathcal{Z}_{\alpha, s}\left(M_{\frac{n}{\alpha}} T_{\alpha k} \chi_{[0, \alpha)}\right)(t, \omega) & =e^{2 \pi i\left(s \frac{k}{\alpha}+\frac{n}{\alpha}\right) t} e^{-2 \pi i \alpha k \omega} e^{-\pi i s k^{2}} \mathcal{Z}_{\alpha, s} \chi_{[0, \alpha)}(t, \omega) \\
& =e^{2 \pi i\left(s \frac{k}{\alpha}+\frac{n}{\alpha}\right) t} e^{-2 \pi i \alpha k \omega} e^{\pi i s k^{2}}
\end{aligned}
$$

The last equality holds since $\mathcal{Z}_{\alpha, s} \chi_{[0, \alpha)}(t, \omega)=1$ for a.e. $(t, \omega) \in \mathbf{S}\left([0, \alpha) \times\left[0, \frac{1}{\alpha}\right)\right)$.

By a change of variable one can show that $\left\{e^{2 \pi i\left(s \frac{k}{\alpha}+\frac{n}{\alpha}\right) t} e^{-2 \pi i \alpha k \omega} e^{-\pi i s k^{2}}\right\}_{n, k}=$ $\left\{e_{n k}\right\}_{n, k}$ forms an orthonormal basis in $L^{2}\left(\mathbf{S}\left([0, \alpha) \times\left[0, \frac{1}{\alpha}\right)\right)\right)$;

$$
\begin{aligned}
& \left\langle e_{n, k}, e_{m, l}\right\rangle_{L^{2}\left(\mathbf{S}\left([0, \alpha) \times\left[0, \frac{1}{\alpha}\right)\right)\right)} \\
& \quad=e^{\pi i s\left(l^{2}-k^{2}\right)} \int_{\mathbf{S}\left([0, \alpha) \times\left[0, \frac{1}{\alpha}\right)\right)} e^{2 \pi i\left(s \frac{k}{\alpha}+\frac{n}{\alpha}\right) t} e^{-2 \pi i \alpha k \omega} e^{-2 \pi i\left(s \frac{l}{\alpha}+\frac{m}{\alpha}\right) t} e^{2 \pi i \alpha l \omega} d t d \omega \\
& \quad=e^{\pi i s\left(l^{2}-k^{2}\right)} \delta_{l k} \delta_{n, m} .
\end{aligned}
$$

The modified Zak-transform $\mathcal{Z}_{\alpha, s}$ is therefore unitary. Since the fractional Fourier-transform is unitary, and unitarity is preserved by composition, it follows that also $\mathcal{Z}_{\alpha, s, \theta}$ is unitary. 